\title{
Faciliter et accélérer les actions de valorisation des universités par la mise en réseau
}

\author{
Facilitating and accelerating actions in knowledge and technology \\ transfer from universities through networking
}

\author{
Olivier Vande Vyver ${ }^{1}$ \\ ${ }^{1}$ Réseau LiEU, Belgique, director@reseaulieu.be
}

\begin{abstract}
RÉSUMÉ. Au sein d'une petite et moyenne université, par son volume de recherche plus réduit, la valorisation de la recherche visera des objectifs plus larges que celui associé au seul transfert de technologies. II s'agira également de contribuer au développement socio-économique de la région par la mobilisation et le transfert de connaissances via la formation dispensée par les institutions et la collaboration avec les entreprises proches de l'université.

A titre d'exemple, les Universités de la fédération Wallonie-Bruxelles ont décidé de coordonner leurs efforts à travers un réseau (réseau LiEU - Liaison Entreprises Universités) dans le domaine de la valorisation pour mener à bien des projets d'envergure et mutualiser leurs moyens.

Dans cet article, à travers cet exemple et sur base d'une expérience dans le domaine de plus de 15 ans, on trouvera les clés de réussites qui auront permis aux universités de travailler ensemble sur le sujet de la valorisation de la recherche. ABSTRACT. In a small and medium-sized university, with a smaller volume of research, the knowledge transfer activities are not restricted to technology transfer alone. The aim is to contribute to the socio-economic development of the region via training and collaboration with companies close to the university.

As an example, the Universities of 'Fédération Wallonie-Bruxelles' have decided to coordinate their efforts through a network (LiEU network - Liaison Entreprises Universités) in the field of knowledge transfer whenever the collaboration brings a real added value and economies of scale.

In this article, through this example and on the basis of more than 15 years of experience, we will present the keys to success in order to carry out major common projects and pool resources for knowledge transfer activities.

MOTS-CLÉS. valorisation, mobilisation des connaissances, réseau, impact socio-économique, qualité.

KEYWORDS. knowledge transfer, network, collaboration, regional development, quality.
\end{abstract}

\section{Introduction}

La valorisation de la recherche universitaire contribue à la mission de service à la société des universités. La réalisation de cette mission va dépendre de différents facteurs dont la taille de l'université (c'est-à-dire son volume de recherche) et son ancrage dans l'écosystème local. Ce dernier pourra par exemple favoriser le transfert de connaissances à travers des opérateurs publics ou privés tels que des incubateurs, des parcs scientifiques et technologiques.

Les actions associées à la valorisation de la recherche sont liées à l'impact sur la société et plus particulièrement sur l'écosystème local attachée à l'institution universitaire. L'impact ne sera pas que technologique et se traduira de manière plus générale par une mobilisation des connaissances au profit de la société.

Le rôle d'une cellule de valorisation sera donc d'être l'interface entre le monde de la recherche universitaire et la société dans son ensemble. Pour gérer cette acculturation, une structure dédiée à la valorisation (cellule de valorisation ou KTO - Knowledge Transfer Office) pourra être mise en place. Son organisation va dépendre notamment de la taille de l'université.

Une approche de mutualisation des efforts dans ce domaine pourra se faire au profit des institutions universitaires et de l'écosystème socio-économique d'une même région. Compte tenu d'une 
concurrence légitime entre ces différentes institutions, sur quels critères les efforts pourront être mis en commun et pour quelles missions?

Nous illustrerons dans cet article les avantages de travailler en réseau pour des institutions de tailles variables à travers l'exemple du Réseau LiEU' ${ }^{1}$.

\section{Un peu d'histoire...}

\subsection{Le contexte régional}

En décembre 1998, la Région wallonne lançait le projet «Promothée », s'inscrivant dans le programme RIS (Regional Innovation Strategy) de l'Union européenne. Cette action, d'une durée de deux ans, avait pour but de définir, avec l'ensemble des acteurs, une politique wallonne de l'innovation et d'identifier les réformes et les actions prioritaires à mettre en œuvre. Dans ce cadre, un groupe de travail a été chargé de faire des propositions utiles pour mobiliser au mieux le potentiel de recherche universitaire en faveur du développement économique régional incluant l'exploitation des résultats de la recherche (transfert de technologie et création d'entreprise) [PRO 00]. Une des recommandations de ce groupe de travail a été de renforcer les structures de support à la valorisation et de les organiser en réseau.

Le Conseil des Recteurs $\left(\mathrm{CReF}^{2}\right)$ décide de créer un Groupe de Travail interuniversitaire permanent, pour échanger les bonnes pratiques en matière de recherche appliquée et de valorisation. En 2002, ce groupe de travail est confirmé dans ses missions sous la forme d'un réseau : le Réseau LiEU. A partir de 2006, le Réseau LiEU est soutenu par la Région wallonne via le financement d'une cellule de coordination.

Dans le même temps, depuis le 1er janvier 1998, les universités bénéficiaires d'une subvention wallonne pour la recherche sont propriétaires des résultats et des droits générés par cette recherche [DEC 97]. Cette responsabilité en matière de gestion de la propriété intellectuelle, amènera les universités à développer progressivement des activités liées à la valorisation de la recherche. La Wallonie a depuis cette période apporté son soutien dans cette démarche à travers des subventions spécifiques pour la protection des résultats de recherche (Fonds brevets) et pour leur valorisation (Fonds experts). En 2007, le projet MIRVAL ${ }^{3}$ (Mise en Réseau de la Valorisation) permettra aux KTO d'assurer pleinement leurs missions en matière de valorisation de la recherche. Ce projet sera prolongé en 2014 via le projet MIRVAL+.

\subsection{Un réseau de KTO}

Le réseau LiEU (Liaison Entreprises Universités) relève directement du Conseil des Recteurs des Universités Francophones de Belgique ( $\mathrm{CReF})$. Les membres du réseau LiEU sont les cellules de valorisation (KTO) des universités. Ces KTO travaillent ensemble au sein du Réseau LiEU tout en conservant leur autonomie. Leur mission étant de valoriser les résultats de la recherche des institutions universitaires. Le nombre de chercheurs au sein de chaque institution varie de 800 à 3.000 environ pour un total de plus de 10.000 chercheurs [CRE 14].

\footnotetext{
${ }^{1}$ Pour plus d'informations sur le Réseau LIEU : https://www.reseaulieu.be/ - contact@reseaulieu.be

${ }^{2}$ Le CReF (Conseil des Recteurs Francophones): http://www.cref.be/

${ }^{3}$ Projet MIRVAL - Mise en réseau de la valorisation. Financement dans le cadre de la programmation 2007-2013 (FSE),

cofinancement par la Wallonie. Plus d'informations sur : https://www.enmieux.be/
} 


\section{Le processus de valorisation}

Envisager une mutualisation de certaines actions en matière de valorisation de la recherche requiert que les acteurs de cette mutualisation présentent une cohérence en matière de stratégie de valorisation et des moyens qui y sont affectés.

Il est donc indispensable de pouvoir s'accorder sur la définition des missions associées à la valorisation de la recherche et d'en déduire ses indicateurs d'impact sur la société.

\subsection{Les indicateurs d'impact de la valorisation de la recherche}

Du fait de sa nature, les indicateurs d'impact de la valorisation de la recherche sur la société dépassent le cadre des actions seules menées par le KTO. En effet, la valorisation de la recherche nécessite un partenariat entre le chercheur (le porteur de projet), l'acteur qui va absorber le résultat de la recherche (entreprise, association...), l'équipe chargée de valorisation au sein du KTO et d'autres partenaires nécessaires (incubateurs, investisseurs...). Chacun remplissant sa mission au profit d'un projet commun.

L'implication et l'engagement de ces acteurs va dépendre de l'état de maturation du résultat. L'échelle TRL (Technology Readiness Level) permet de positionner une technologie (et donc sa maturité) tout en mesurant les efforts nécessaires et les partenariats indispensables pour arriver à sa commercialisation. L'idéal étant d'élargir ce concept pour intégrer d'autres notions que celle uniquement technologique, pour évaluer la maturité du transfert d'une idée issue d'un laboratoire. Le travail réalisé sur l'échelle IRL (Innovation Readiness Level) est un bon exemple de cet élargissement [GIL 17]. Sur cette base, il est possible de relier les actions propres du KTO et les résultats attendus dans le processus de valorisation et d'en déduire des indicateurs de résultats.

Les indicateurs communément admis pour la valorisation de la recherche sont (ASTP $\left.{ }^{4}, \mathrm{CURIE}^{5} \ldots\right)$ les suivants :

- Relatif à la gestion de la PI

- Nombre de déclaration d'invention,

- Nombre de demande de brevets

- Nombre de brevets délivrés

- Relatif aux transferts de technologie

- Nombre de licences

- Nombre de spin-offs

A cela s'ajoute en fonction des cas, des indicateurs relatifs à la collaboration menée entre l'université et le monde socio-économique :

- Nombre de contrats de collaboration avec une entreprise

Il est intéressant de pouvoir bien définir ce qu'on entend par entreprise et la concrétisation effective de cette collaboration : prestations de service, accord de parrainage pour un projet de recherche, accord de consortium dans le cadre d'un projet de recherche. Pour chacune de ces situations, les actions attendues des KTO seront différentes.

Des indicateurs financiers sont également repris :

\footnotetext{
${ }^{4}$ ASTP : Association Européenne des professionnels du transfert de technologie https://www.astp4kt.eu/

${ }^{5}$ CURIE : Association française des professionnels du transfert de technologie et de connaissance : $\underline{\text { https://www.curie.asso.fr/ }}$ 
- Montant des royalties, des revenus de licence

- Montant des conventions de recherche

Il est néanmoins nécessaire d'être prudent quant à l'utilisation des indicateurs de ce type pour illustrer les missions de valorisation de la recherche. Ils ne tiennent pas compte de l'impact des missions principales des universités à savoir la transmission et la création de connaissances au profit de la société. De plus, en matière de comparaison entre KTO, ils ne tiennent pas compte de la taille de l'université et des disciplines de recherche qui y sont menées. Enfin, ces indicateurs classiques en matière de valorisation de la recherche font intervenir différentes actions à différents moments suivant le processus de valorisation du montage d'un projet de recherche au transfert du résultat comme nous le verrons ci-dessous.

\subsection{Le processus de valorisation}

En fonction de la taille de l'université et de sa stratégie en matière de valorisation, certaines actions vont revêtir plus d'importances que d'autres. La stratégie décidée et mise en place au sein de l'institution universitaire permettra d'offrir un cadre clair de fonctionnement pour la valorisation de la recherche.

Généralement, l'action sur le financement de la recherche et le support apporté aux chercheurs en matière de montage de projet et de partenariats seront favorisés étant donné qu'il s'agit d'une première étape cruciale de production de connaissance pouvant par la suite être valorisée.

Cette stratégie évoluera également suivant trois axes traduisant la maturité acquise par l'institution universitaire en matière de valorisation de la recherche [SCA 18] :

- L'expérience des membres du KTO

- Le type et l'intensité des relations de l'université avec la société et plus particulièrement avec le tissu industriel proche

-L'organisation de l'institution (et sa taille) et l'intégration en son sein des activités de valorisation de la recherche

La mutualisation ou de manière plus générale, le partenariat entre structures de valorisation (KTO) va dépendre de la position qu'ils occupent sur ces différents axes. Par exemple, pour des KTO jeunes (c-à-d avec une faible expérience), des actions communes en matière de formation pourront être mise en place.

Cependant, même avec des maturités différentes, le processus de valorisation peut s'envisager de la même manière. A titre d'exemple, à la figure 1, nous retrouvons le processus de valorisation au niveau du réseau LiEU en considérant quatre catégories d'actions :

-Financement :

- Détection :

-Maturation :

- Transfert : 


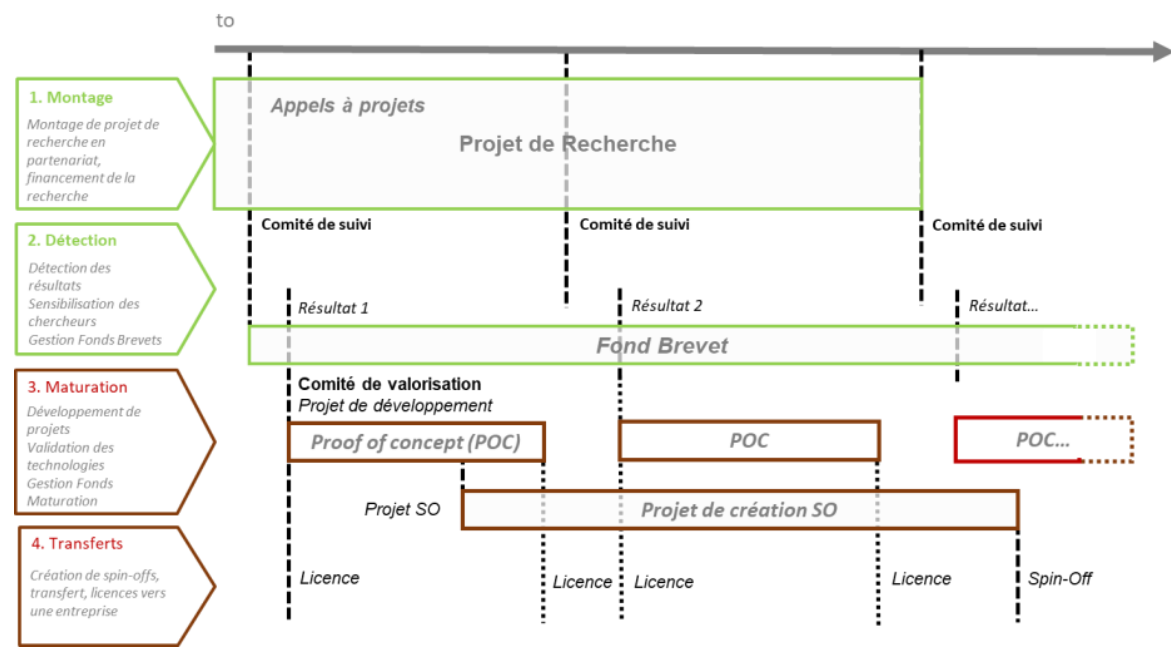

Figure 1. Processus de valorisation au cours du temps (Source: auteur, LiEU)

\section{La mutualisation au profit du processus de valorisation}

La mutualisation des efforts entre institutions en matière de valorisation de la recherche demandera une harmonisation entre les processus de valorisation, les objectifs attendus des actions et les indicateurs d'impact mesurés.

Concrètement, comment arriver à une harmonisation du processus de valorisation entre institutions universitaires d'une même région et de tailles différentes?

\subsection{Professionnalisation du processus de valorisation de la recherche}

En 2007, les institutions du réseau LiEU ont été les bénéficiaires d'un projet commun sur la valorisation de la recherche : projet MIRVAL $^{6}$. Le projet prévoyait une série d'actions mutualisées aux profits des chercheurs et des entreprises de la Wallonie.

Parmi les axes du projet, un axe portait spécifiquement sur la mise en place d'une démarche qualité pour l'organisation de la valorisation de la recherche [VAN 19]. Si l'objectif n'était pas d'avoir une certification de type ISO9001, il n'en demeurait pas moins qu'il était important d'avoir un engagement d'un service de qualité auprès de nos clients : les chercheurs et les entreprises.

L'ensemble des KTO se sont mis d'accords pour harmoniser leur processus de valorisation avec comme objectif pour chacun de :

- Augmenter l'efficacité envers ses différents bénéficiaires

- Garantir le professionnalisme des activités

- Assurer le développement et la pérennité des structures de valorisation

- Capitaliser sur l'expérience et la connaissance des membres

-Simplifier le travail quotidien

- Faciliter l'évolution des modes de fonctionnement et l'agilité

- Conférer une meilleure visibilité des services envers les bénéficiaires

En 2011, un référentiel qualité a été réalisé. Ce référentiel formalise les engagements et exigences souhaités par les membres du Réseau LiEU tout en tenant compte des demandes des différents

\footnotetext{
${ }^{6}$ Projet MIRVAL - Mise en réseau de la valorisation. Financement dans le cadre de la programmation 2007-2013 (FSE), cofinancement par la Wallonie. Plus d'informations sur : https://www.enmieux.be/
} 
bénéficiaires : l'institution, les chercheurs de l'institution, les entreprises privées et publiques, le réseau LiEU et ses membres, le personnel des KTO, les pouvoirs publics, les acteurs de l'innovation (Région).

D'un point de vue pratique, ce référentiel s'est concrétisé par une charte qualité reprenant les engagements en matière de valorisation de la recherche. Il est intéressant de noter que cette démarche qualité commune ne dépend pas de la taille des institutions. Il est possible d'avoir un processus commun qui réponde aux attentes de chacun. La différence reposera sur les moyens disponibles et mis en place pour les différentes actions prévues.

Ceci aura donc permis d'harmoniser notre processus de valorisation tel que présenté à la figure 1 .

Cette démarche qualité aura apporté aux KTO :

- Un processus de valorisation commun avec des outils pour l'implémentation des actions ;

- La mise en place et l'identification d'indicateurs de réalisations et de résultats pour un suivi des activités.

Au niveau de nos cibles, les chercheurs et les entreprises, cela aura permis de clarifier et préciser les services offerts par les KTO et d'avoir ainsi une communication cohérente et efficace.

Le processus de valorisation, les objectifs attendus et les indicateurs étant clairement établis pour chacune des institutions, il est plus facile d'envisager une mutualisation des efforts sur certaines actions.

\subsection{La mutualisation en réponse aux besoins des chercheurs et des entreprises}

Pour nos bailleurs de fonds, la priorité était de pouvoir s'assurer que le financement apporté aux KTO (notamment à travers les projet MIRVAL et MIRVAL+) ne fasse pas l'objet d'une dispersion des efforts. Il a nous donc été logiquement demandé de nous coordonner pour répondre aux besoins des entreprises en matière de collaboration avec les universités. De manière équivalente, pour nos institutions, il était important d'assurer un support aux chercheurs en matière de valorisation de leurs résultats de recherche.

La première étape a été d'identifier de manière précise les besoins des chercheurs et des entreprises. Pour ce faire, deux groupes de travail ont été mis en place au sein du réseau LiEU. Chacun des groupes étant constitué d'une personne coordinatrice, membre d'un KTO avec une équipe constituée de personnes issues des autres KTO du réseau.

\subsubsection{Quelles sont les attentes des chercheurs vis-à-vis de sa cellule de valorisation?}

L'objectif était de pouvoir répondre aux besoins des chercheurs en matière de valorisation des résultats de leur recherche en développant des outils de support adéquats.

Pour ce faire, un questionnaire commun a été élaboré en vue de rencontrer les chercheurs des institutions. Ces rencontres personnalisées auront permis d'identifier les besoins des chercheurs :

- Avoir un seul interlocuteur et un accompagnement personnalisé :

Ce besoin peut être facilement rencontré à partir du moment où un suivi des laboratoires est organisé au sein du KTO. Une personne de contact permanente pour le KTO et peu importe les sujets traités sera d'une grande aide pour les chercheurs. De plus, cela permettra de pouvoir mettre en place une relation de confiance facilitant ultérieurement la détection des résultats de la recherche.

La piste de mutualisation envisagée pour répondre à ce besoin a été de pouvoir assurer l'accompagnement de projets collaboratifs impliquant différentes institutions. Compte tenu du volume de recherche, il est impossible pour un KTO de pouvoir assurer un suivi complet et efficace de 
l'ensemble des projets de ses chercheurs. La solution proposée a été de pouvoir se répartir les tâches en identifiant un « référent valorisation » qui sera mandaté par l'ensemble des institutions pour le suivi de certains projets collaboratifs. Pour des petites institutions, cela permet aussi de pouvoir compter sur l'expertise d'un autre KTO dans différents domaines.

- Pouvoir conseiller le chercheur par rapport aux outils de financement de la recherche, les domaines et thématiques stratégiques liés à ces financements :

Pour un chercheur, pouvoir compter sur des informations claires en matière d'appel à projets, est fondamental pour le financement de sa recherche. Un support du KTO au montage des projets permettra de donner toutes les chances de réussites du projet en matière d'évaluation de l'impact du projet de recherche sur la société.

$\mathrm{Au}$ niveau du réseau, il est possible pour ce point d'envisager une centralisation des informations liées aux appels à projets et de pouvoir fournir de manière claire et précise ces informations aux chercheurs des institutions universitaires. D'un point de vue juridique, il est possible d'avoir des modèles d'accords communs répondant aux critères des bailleurs de fonds et traduisant une position commune des institutions universitaires.

-Faciliter la recherche de partenariats industriels et académiques :

Ce besoin est en lien avec le précédent. Dans le cadre de certains appels à projets, les conditions d'éligibilité demandent des partenariats académiques et industriels. La promotion des expertises et des équipements en vue de pouvoir trouver des partenariats de recherche prend tout son sens dans ce contexte.

Il est donc utile pour les KTO de mutualiser les efforts en matière de promotion des compétences des chercheurs et de mise en relation entre les chercheurs et avec les entreprises. Pour des petites institutions, la mutualisation dans cette matière est directement bénéfique compte tenu de l'investissement que cela demande en matière d'organisation d'évènements par exemple.

-Se former pour mieux appréhender la valorisation dans sa globalité et les différentes étapes qui la composent :

Le processus de valorisation de la recherche peut paraître obscur et complexe pour des chercheurs qui ne sont pas coutumier du fait. Il est donc important de pouvoir facilement et rapidement rassurer les chercheurs et leur apporter les réponses aux questions qu'ils se posent sur ce sujet.

La mise en place d'outils de sensibilisation et de formation peut facilement s'envisager de manière concertée.

\subsubsection{Quelles sont les attentes des entreprises vis-à-vis des cellules de valorisation ?}

De manière similaire que pour les chercheurs, un groupe de travail a été mis en place avec comme objectif de pouvoir identifier les besoins des entreprises de notre région. La particularité de cette action repose sur la collaboration du réseau LiEU avec l'Union Wallonne des Entreprises ${ }^{7}$ par la mise en place d'un groupe de travail mixte constitué de représentants des entreprises grandes et petites, des fédérations et des Pôles de compétitivité, et des représentants des KTO du réseau.

Le principal objectif pour les KTO était de pouvoir renforcer leur rôle dans le développement économique de la région en captant les besoins d'innovations des entreprises. Au-delà de ce premier objectif, le travail aura également permis de clarifier et de mieux préciser les services offerts par les KTO aux entreprises.

\footnotetext{
${ }^{7}$ Union Wallonne des entreprises (UWE) : https://www.uwe.be/
} 
En effet, la première constatation était une méconnaissance des services offerts par les KTO et un sentiment de complexification de ces services. Ce travail aura donc eu une incidence importante sur la communication à l'extérieur des services offerts par les KTO et du réseau LiEU.

Les attentes principales des entreprises étaient :

- Une simplification du message. Plus précisément, en termes de communication vers l'extérieur, proposer une clarification et une simplification de la présentation des services offerts par le réseau LIEU et les KTO.

- Des portes d'entrées sectorielles. Afin de ne pas faire le tour des institutions à la recherche du laboratoire pouvant répondre à leur demande, le besoin des entreprises était d'avoir un accompagnement de la demande et un relais vers l'ensemble des laboratoires.

\subsection{Une organisation du réseau de KTO pour mutualiser les ressources et les moyens}

Sur base des résultats de ces sondages auprès des chercheurs et des entreprises, le réseau LiEU a décidé d'initier une série d'actions afin de répondre aux besoins de ses cibles.

La première concernait la promotion concertée des expertises et des compétences de nos laboratoires. Le défi aura été de pouvoir proposer aux entreprises de nos régions des offres de compétences et expertises pertinentes de l'ensemble de nos laboratoires. Rappelons également que du point de vue des entreprises, la demande principale était de pouvoir s'appuyer sur une personne de contact pouvant assurer le lien et le relais vers l'ensemble des laboratoires des universités de notre région. Il a été d'abord nécessaire de se mettre d'accord sur les thématiques prioritaires pour lesquels nous allions réaliser ces actions de promotions. Le choix s'est porté tout naturellement sur les axes stratégiques des Pôles de compétitivité de la Wallonie. Ces derniers à travers des appels à projets, soutiennent des projets de recherche collaboratifs impliquant plusieurs entités de recherche et d'entreprises ${ }^{8}$.

Le réseau LiEU s'est organisé en différents groupes de travail thématiques (GTT) couvrant les domaines des pôles de compétitivité : bio-tech santé, matériaux, agro-tech, énergie et environnement, TIC. Un groupe de travail supplémentaire et transversal a été mise en place ultérieurement et portant sur les Sciences Humaines et sociales. Chaque GTT est constitué de membres des KTO spécialistes du domaine et maitrisant bien la recherche de son institution dans ce domaine. Un coordinateur est désigné et consacre donc une partie de son temps au profit du réseau. Un équilibre institutionnel entre les coordinateurs permet de se partager les tâches entre institutions. L'ensemble de l'organigramme faisant l'objet d'un accord par le comité de gestion de LiEU.

$\mathrm{Au}$ sein de ces GTT, nous retrouvons aussi les personnes de référence de chaque Pôles de compétitivité associé à la thématique. Ceci afin d'assurer un lien direct mais non exclusif avec les membres industriels des Pôles (voir figure 2).

Les missions de ces GTT sont les suivantes :

- Accompagner et suivre les demandes externes de compétences

- Traiter et partager l'expérience des membres du GTT sur des problématiques et des questions rencontrées au sein des KTO dans la thématique du GTT

- Promouvoir de manière concertée les compétences et les expertises de nos laboratoires

-Rencontrer les entreprises, fédérations et Pôles du secteur afin de faciliter l'identification de leurs besoins

\footnotetext{
${ }^{8}$ Pôles de compétitivité de la Wallonie : https://clusters.wallonie.be
} 
Au-delà de ces missions, ces GTT présentent des portes d'entrée sectorielle pour les entreprises souhaitant entrer en contact avec les expertises de nos chercheurs. Le coordinateur du GTT étant la personne de contact principale.

A côté de ces groupes thématiques, des groupes de supports transversaux ont été également mis en place sur les domaines suivants :

- Service aux chercheurs, en charge des projets en matière de sensibilisation et de formations

- Juridique en charge de pouvoir proposer des modèles d'accords juridique de manière concerté entre les universités

- Communication en charge des projets de promotions

-Qualité et professionnalisation en charge de la mise en place d'actions professionnalisantes pour les membres du réseau LiEU.

Afin d'assurer une coordination et un suivi de ces actions, la Wallonie soutient financièrement la coordination du réseau LiEU. Enfin, l'ensemble des actions communes fait l'objet d'un passage en revue mensuel au sein du comité de gestion LiEU constitué des responsables des KTO.

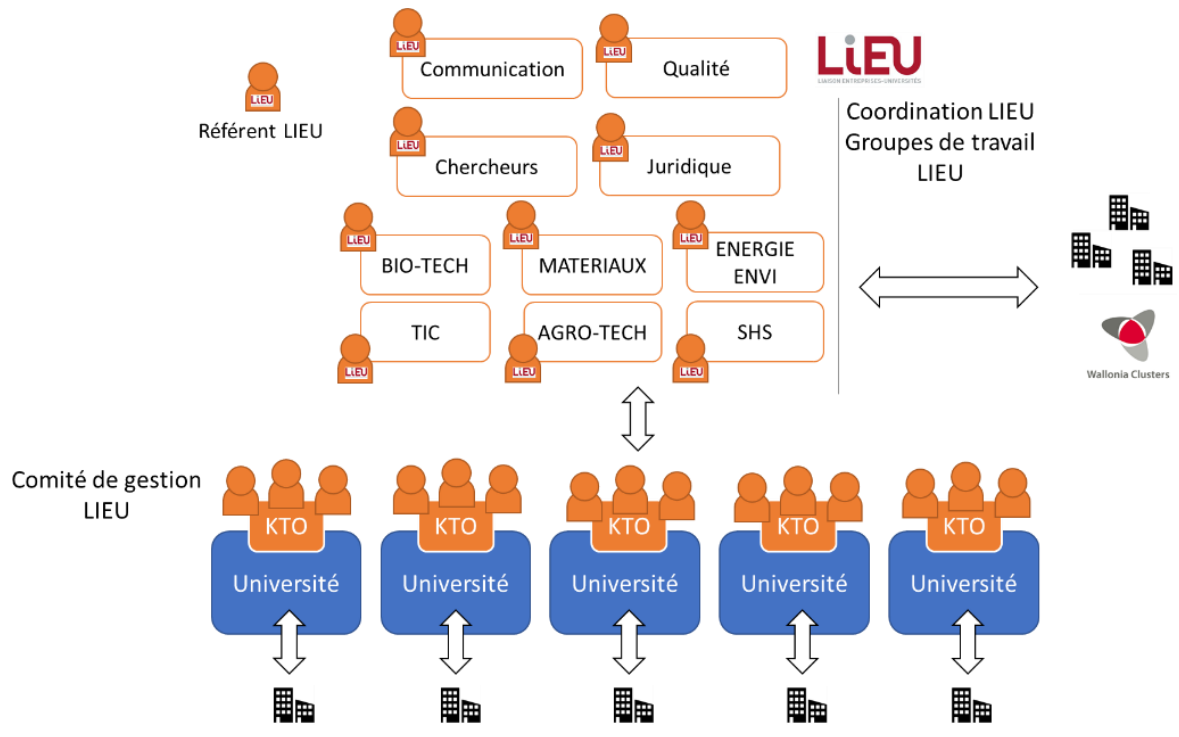

Figure 2. organisation en réseau de KTO - illustration du réseau LiEU - modèle 2020 (source : auteur, LIEU).

A ce jour, les KTO ont pu établir des liens de proximité et de confiance avec les chercheurs et les entreprises pour un transfert de connaissances et de technologies efficace et rapide.

En 2021, le modèle du réseau LiEU évoluera afin de répondre aux défis de notre société en étant plus agile et en permettant une meilleure et efficace mutualisation des ressources et des moyens. L'organisation des GTT LiEU évoluera donc vers des groupes-projet composés d' « experts LiEU ». En fonction des besoins et des sollicitations de nos cibles, ces personnes expertes pourront être mobilisées au profit de l'ensemble des KTO. De plus, des «référents LiEU » assureront les contacts externes vers nos partenaires et entreprises et assureront le relais vers les membres de LiEU.

L'évolution naturelle de cette organisation repose sur un climat de confiance entre les membres du réseau qui à travers des actions communes sont parvenus à remplir également leurs objectifs au niveau du KTO local. 


\section{Illustrations de mutualisation en matière de valorisation de la recherche}

Cette organisation d'un réseau de KTO qui repose sur une mise à disposition de personnel de chaque KTO, permet de mener des projets communs en mutualisant les forces et les ressources. On trouvera ci-après quelques exemples de projets développés au sein du réseau LiEU et qui peut être transposable à d'autres réseaux ou associations de KTO.

\subsection{Mise en place d'un inventaire de compétences commun et accessible}

Dans un premier temps, un travail de mutualisation peut être mis en place pour la promotion des compétences et des expertises des laboratoires des universités.

Pour les entreprises, il est important d'avoir une information lisible et précise des offres des laboratoires à travers une «fiche de compétences ». Celle-ci doit être orientée en réponse à un besoin du marché et dans une perspective de solution à offrir.

Quelles sont les informations utiles pour une entreprise ? Cette question a été posée à un échantillon d'entreprises innovantes de la région de manière à se mettre d'accord sur un canevas de fiches de compétences. Les informations utiles en plus des expertises du laboratoire étaient de connaître les sujets de recherche en cours, les équipements accessibles, les offres de technologie et les offres de formations. Un site internet permettant un accès à l'ensemble des fiches a été développé. Au niveau du réseau LiEU, cela représentait un peu plus de 500 fiches de compétences et 70 offres de technologie en ligne. Cette activité demande cependant un investissement pouvant être important notamment en ce qui concerne la mise à jour des fiches de compétences.

Pour les KTO, les avantages sont :

- De pouvoir réaliser une promotion des compétences consolidées et mises à jour. Ce point est important pour l'organisation des évènements de promotion ;

- D’avoir une connaissance plus approfondie des expertises disponibles au sein des laboratoires ;

-De pouvoir identifier des opportunités de collaborations et des complémentarités entre les laboratoires.

Au niveau du réseau LiEU, étant donné le faible nombre de vue sur le site, il a été décidé après 5 ans d'inventaire exhaustif d'arrêter de consolider et de mettre à jour les fiches de compétences. Néanmoins, afin de répondre aux besoins d'informations des entreprises, nous avons privilégié des inventaires personnalisés à la demande des entreprises ou de nos pôles de compétitivité. Notre site internet met en avant ces portes d'entrée sectorielle. Actuellement, nous réalisons à la demande près de 60 inventaires personnalisés par an, cartographiant ainsi un domaine technologique. Ces informations sont à disposition de nos entreprises, pôles et clusters.

L'inventaire de compétences présentera un intérêt majeur s'il est possible de mettre en relation l'ensemble des acteurs de l'innovation et les entreprises. Cela peut se faire par une mise en commun des bases de données de différents opérateurs pouvant de cette manière faire apparaitre des filières technologiques. De cette manière, la mise en relation entre les entreprises, les chercheurs et autres acteurs d'innovation peut être facilité et encouragé à l'image de ce qui se fait à travers la plateforme $\mathrm{CRAFT}^{9}$ et l'outil Plug in Labs ${ }^{10}$ des universités et société de valorisation ou encore de Digital Wallonia $^{11}$ en Wallonie.

\footnotetext{
${ }^{9}$ Plateforme CRAFT : $\underline{\text { https://www.platform-craft.eu/ }}$

${ }^{10}$ Plug In Labs : https://www.pluginlabs.fr/

${ }^{11}$ Digital Wallonia : https://www.digitalwallonia.be/fr
} 


\subsection{Organisation d'évènements de rencontre chercheurs-entreprises}

Pour les entreprises, il y a clairement une plus-value de pouvoir proposer en un seul lieu et moment des rencontres avec les chercheurs de l'ensemble de nos institutions autour d'une problématique industrielle identifiée.

La concurrence entre les institutions ne doit pas constituer un frein à l'organisation de ces évènements mais au contraire être une opportunité de pouvoir montrer dans une thématique donnée les complémentarités entre les chercheurs. Ce travail important d'identification mené au sein du réseau (via les GTT) apporte une grande valeur ajoutée pour les entreprises et les chercheurs.

$\mathrm{Au}$ niveau du réseau LiEU, le concept de Lab'Insight ${ }^{12}$ a été développé et mis en place. Il s'agit d'événements visant à faire émerger des projets d'innovation entre entreprises et chercheurs rassemblés autour d'une thématique répondant à un besoin industriel très ciblé (ex. : la conservation des aliments réfrigérés, le stockage d'énergie...). Ils permettent la promotion des compétences des laboratoires universitaires, en veillant à offrir aux entreprises une offre complémentaire couvrant les différents aspects liés à leur préoccupation, structurée et consolidée à l'échelle du réseau (en présentant les compétences de l'ensemble des universités membres).

Cette activité demande un investissement humain et matériel important en matière d'organisation et de préparation. En mutualisant au sein du réseau, il est possible de se répartir les efforts et les ressources tout au long de l'année et proposer aux entreprises un programme de rencontre couvrant différentes thématiques.

Cette action de rencontre chercheurs-entreprise n'est pas à négliger dans le cadre des missions de la valorisation de la recherche. Même si l'impact direct de celle-ci n'est pas évident et facile à mesurer. A ce sujet, on se réfèrera au travail du Prof. Jonsson [JON 15] qui montre l'importance pour une université d'avoir une stratégie de stimulation des échanges entre entreprises et chercheurs afin de faciliter le transfert de connaissances. A travers ces rencontres, la confiance qui s'installe entre les acteurs va permettre à partir des premiers contacts, de conduire ultérieurement à une collaboration effective. Il est cependant important également d'impliquer dans ces rencontres l'ensemble des acteurs de l'innovation de la région : centres de recherche, entreprises, fédération et pôles de compétitivité.

Par la suite, la mutualisation prend également tout son sens en matière de suivi et d'accompagnement des rencontres établies après cette première mise en relation.

\subsection{Un outil de sensibilisation à la valorisation de la recherche}

Pour répondre aux besoins des chercheurs en matière de sensibilisation à la valorisation de la recherche, des formations et des outils ont été développés.

Deux axes de travail ont été identifiés. Le premier portait sur l'organisation de séances de formation/sensibilisation des chercheurs concrétisée par l'élaboration d'un matériel commun de formation par le réseau LiEU. Le second axe portait sur l'élaboration d'un outil pédagogique et interactif à l'attention des chercheurs, présentant et expliquant les étapes de la valorisation (figure 3$)^{13}$.

\footnotetext{
${ }^{12}$ Lab'Insight : pour plus d'informations sur le concept Lab'Insight (https://www.reseaulieu.be - contact@reseaulieu.be ) et sur la chaine Youtube du Réseau LIEU.

${ }^{13}$ Outil de sensibilisation à la valorisation de la recherche développé par le réseau LIEU : https://proprieteintellectuelle.reseaulieu.be/
} 


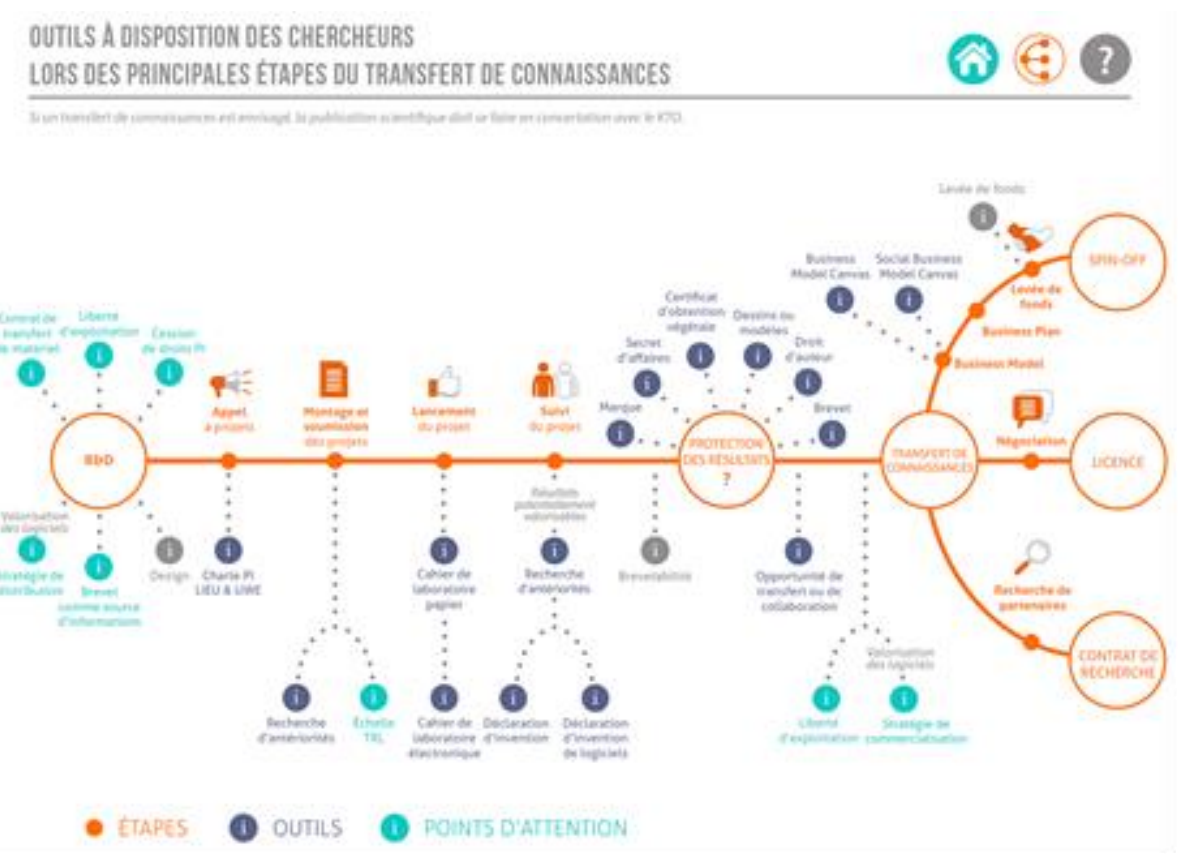

Figure 3. outil pédagogique et interactif présentant et expliquant le processus de valorisation (page de présentation) - OLIEU

Cet outil a fait l'objet d'une présentation dans le cadre du Congrès CURIE 2017 lors de l'atelier «Les nouvelles formes de sensibilisation et d'incitation des chercheurs et des doctorants » et montrait l'importance de ce genre d'outil en matière de communication auprès des chercheurs [DEB 17].

La mutualisation trouve tout son intérêt dans cette démarche afin de pouvoir réaliser des documents explicatifs et synthétiques sur des sujets pointus. En effet, cela permet de s'appuyer sur les expertises présentes au sein des KTO et permettre ainsi à chacun d'apporter sa contribution à un outil commun.

\subsection{Une charte de collaboration avec les entreprises}

Pour les bailleurs de fonds finançant des projets de recherche collaboratifs impliquant au minimum une entreprise et une université, il est indispensable de pouvoir compter sur des règles claires et comprises en matière de propriété intellectuelle. Ceci avec comme objectif de pouvoir maximiser les possibilités de valorisation des résultats de recherche, au bénéfice des partenaires tout en tenant compte de la réglementation en matière d'aides d'Etat.

En collaboration avec l'Union Wallonne des Entreprises (UWE), le réseau LiEU a élaboré une charte de la gestion de la PI. Ce document reprend les principes directeurs applicables en matière de propriété et valorisation des résultats issus de recherches effectuées en partenariat entre universités, hautes écoles, centres de recherche et entreprises, dans le cadre de tout projet collaboratif financé par des fonds publics régionaux ${ }^{14}$.

\section{Conclusions}

Chaque institution en matière de valorisation de la recherche au profit de sa Région rencontre les mêmes objectifs. Suivant la stratégie et les actions mises en place dans le cadre de la valorisation de la recherche au sein des institutions, il est possible d'envisager une mutualisation des moyens pour des actions qui ne pourraient pas être mises en œuvre par une seule institution d'autant plus si cette dernière présente un nombre de chercheurs relativement faible.

\footnotetext{
${ }^{14}$ Charte PI : disponible en téléchargement sur le site du réseau LIEU ou sur demande : (https://www.reseaulieu.be/ contact@reseaulieu.be )
} 
Cette mutualisation pourra se traduire par l'association des structures de valorisation à travers, par exemple, la mise en réseau. Les actions communes portées par ce réseau peuvent s'envisager facilement pour les chercheurs en matière de sensibilisation et de formation ; et pour les entreprises en matière de promotion concertées des compétences universitaires et de relais vers l'ensemble des laboratoires d'une même région.

Ce processus se met en place progressivement et la confiance qui s'établit au cours du temps entre les cellules de valorisation amènera des projets plus ambitieux en matière de mutualisation des ressources et des moyens au profit du développement économique régional.

\section{Remerciements et contacts}

L'ensemble des projets mis en place par le réseau LiEU et présenté dans cet article repose sur l'activité et l'investissement des membres du Réseau LiEU, qu'ils en soient tous remerciés ici et plus particulièrement, Mme Fabienne Hocquet (pour le GT entreprise), Mme Christine Guyot (pour le GT Chercheur) et Mme Carole Bastin (pour la coordination LiEU).

Pour de plus amples informations sur le réseau : https://www.reseaulieu.be/ - contact@reseaulieu.be

Les projets MIRVAL, MIRVAL+ mentionnés dans cet article ont été financés par les Fonds FSE et par la Wallonie pour les périodes de programmation (2007-2013 et 2014-2020) ${ }^{15}$

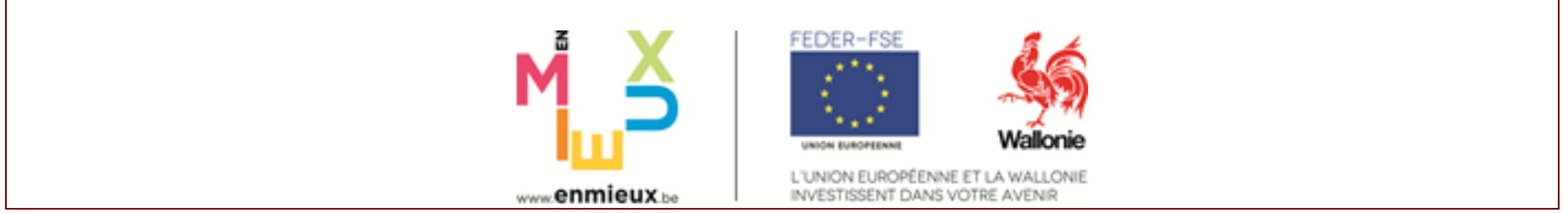

\section{Bibliographie}

[DEB 17] : F.DEBUIGNE,“Mieux communiquer pour mieux valoriser”, Congrès CURIE 2017.

[CRE 14] : CReF - Aperçu statistique sur les étudiants et sur le personnel des universités de la Fédération WallonieBruxelles - $2014^{16}$

[DEC 97] : Décret du 17 décembre 1997. - Décret-programme portant diverses mesures en matière d'impôts, taxes et redevances, de logement, de recherche, d'environnement, de pouvoirs locaux et de transports (entrée en vigueur le $1^{\text {er }}$ janvier 1998)

[GIL 17] : O.GILLIEAUX, F.DOOMS,“L'Innovation Readiness Level (IRL) et autres outils d'idéation; quand l'innovation se fait collaborative", Séminaire Liège Créative, 28 novembre 2017 - Liège ${ }^{17}$

[JON 15] : L.JONSSON, E.BARALDI, L-E.LARSSON, P.FORSBERG, K.SEVERINSSON, "Targeting Academic Engagement in Open Innovation : Tools, Effects and Challenges for University Management", Journal of the Knowledge Economy (2015) $6: 522-550$

[PRO 00] : Rapport final de l'étude PROMETHEE ${ }^{18}$ - Décembre 2000

\footnotetext{
${ }^{15}$ Projet MIRVAL - Mise en réseau de la valorisation. Financement dans le cadre de la programmation 2007-2013 (FSE), cofinancement par la Wallonie. MIRVAL+ - Mise en reseau pour plus de valorisation. Financement dans le cadre de la programmation 2014-2020 (FSE), confinancement par la Wallonie. Plus d'informations sur: https://www.enmieux.be/

${ }^{16}$ http://www.cref.be/annuaires/apercu-historique

${ }^{17}$ https://www.liegecreative.be/evenements/linnovation-readiness-level-irl-et-autres-outils-dideation-quand-linnovation-se-fait

${ }^{18}$ https://recherche-technologie.wallonie.be/fr/menu/archives/archives/programme-promethee.html
} 
[SCA 18] : J.SCANLAN, "A capability maturity framework for knowledge transfer", Industry and Higher Education (2018) - Volume : 32 issues : 4, page(s) : 235-244, 2018

[VAN 19] : O.VANDE VYVER, “La démarche qualité au sein d'un reseau”, Congrès CURIE 2019 